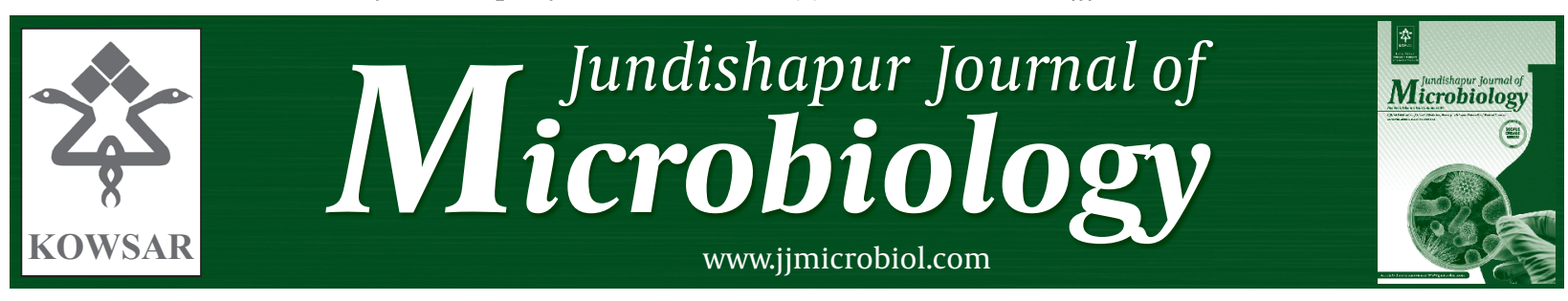

\title{
Serotyping, Antibiotic Susceptibility Pattern and Detection of hlyA Gene Among Cholera Patients in Iran
}

\author{
Manijeh Sedaghat ${ }^{1}$, Fateh Rahimi ${ }^{2}$, Malihe Talebi ${ }^{3}$ Mohammad Reza Pourshafie ${ }^{4^{*}}$ \\ ${ }^{1}$ Department of Microbiology, Pasteur Institute of Iran ,Tehran,IR Iran \\ ${ }^{2}$ Department of Microbiology, Pasteur Institute of Iran, Tehran, IR Iran \\ ${ }^{3}$ Tehran University of Medical Sciences, School of Medicine, Department of Microbiology, Tehran, IR Iran \\ ${ }^{4}$ Department of Microbiology, Pasteur Institute of Iran, Tehran, IR Iran
}

\begin{tabular}{l}
\hline A R T I C L E I N F O \\
\hline Article type: \\
Original Article \\
\hline Article history: \\
Received: 03 Mar 2012 \\
Revised: 15 Apr 2012 \\
Accepted: 18 Apr 2012 \\
\hline
\end{tabular}

Keywords:

Vibrio cholerae

Hemolysin Factors

Polymerase Chain Reaction

\begin{abstract}
A B S T R A C T
Background: Cholera is a severe diarrheal illness caused by Vibrio cholerae, which produces a virulence factor named El Tor hemolysin encoded by the hlyA gene.

objectives: This study meant to analyze the phenotypic characteristics and presence of hlyA gene in $V$. cholerae isolated from patients in Iran. The hlyA gene which codes for hemolysin, plays an essential role in manifestation of cholera ,and could be used to diagnose pathogenic V. cholerae El Tor O1 strains.

Patients and Methods: One hundred stool samples from the patients with cholera during 2002-2003 were collected from Tehran, Kashan, Kermanshah and Ahvaz cities, which were subject to diagnostic tests. Serotyping, and antibiotic susceptibility tests were applied and polymerase chain reaction (PCR) was also used to detect the hlyA gene.

Results: The group specific antisera identified the isolates as Ogawa, Inaba, Hikojima and NAG (Non-agglutinable) in $74 \%, 3 \%, 0 \%$ and $23 \%$ of the isolates, respectively. Antibiotic susceptibility test showed that all of the strains were sensitive to ciprofloxacin, gentamycin and doxycyclin but the isolates showed resistance to sulfamethoxazole/trimethoprim (74\%), erythromycin (64\%) and tetracycline (50\%). V. cholerae El Tor isolates were $100 \%$ positive for hlyA gene, but hemolysis phenotype characteristics were found in $95 \%$ of the cases.

Conclusions:The results indicated that Ogawa serotype was identified as the dominant serotype which revealed multiple antibiotic resistances to sulfamethoxazole/trimethoprim, oxytetracycline, erythromycin, tetracycline and chloramphenicol. The presence of hlyA gene in nonhemolytic strains of $V$. cholerae O1 biotype El Tor indicated that some factors prevent expression of the hemolysin gene.
\end{abstract}

Published by Kowsar Corp, 2013. cc 3.0.

- Implication for health policy/practice/research/medical education:

Even though cholera is easily prevented and treated, it remains life- threatening in Iran , this study indicated the need for continuous recording of antibiotic resistance and status of hlyA production Vibrio cholerae El tor at national level.

- Please cite this paper as:

Sedaghat M, Rahimi F, Talebi M, Pourshafie MR. Serotyping, Antibiotic Susceptibility Pattern and Detection of hlyA Gene Among Cholera Patients in Iran. Jundishapur J Microbiol. 2013;6(1):20-3. DOI:10.5812/jjm.4709

\footnotetext{
* Corresponding author: Mohammad Reza Pourshafie, Department of Microbiology, Pasteur Institute of Iran ,Tehran, IR Iran.Tel/Fax: +98-2166405535

E-mail:pour@pasteur.ac.ir, pour62@yahoo.com
}

DOI:10.5812/jjm.4709

(C) 2013 Ahvaz Jundishapur University of Medical Sciences; Published by Kowsar Corp.

This is an Open Access article distributed under the terms of the Creative Commons Attribution License (http://creativecommons.org/licenses/by/3.0), which permits unrestricted use, distribution, and reproduction in any medium, provided the original work is properly cited. 


\section{Background}

Cholera is a disease unique to humans with sporadic epidemic cases, and seven pandemics have been reported that resulted in thousands of deaths and major changes in socio-economic situations worldwide, these outbreaks indicate that cholera is still among the most prevalent human diseases, especially in developing countries (1-3).

The three most commonly reported serotypes of cholera are caused by $01, \mathrm{O} 139$ and non $\mathrm{O} 1$ - non 0139 serotypes. The O1 strains are divided into two biotypes of classical and El Tor with three serotypes of Ogawa, Inaba and Hikojima (2). In some Asian countries such as Pakistan and Afghanistan the disease has been reported as endemic. In Iran, several outbreaks with a number of mortalities in the recent years have been reported $(4,5)$. The genotypic characteristics of Vibrio cholerae are several toxin genes such as cholera toxin (ctx), zonula occludens toxin (zot), the accessory cholera toxin (ace) and toxin-coregulated pilus (tcpA).

\section{Objectives}

In this study, the serological and antibiotic resistance patterns of the $V$. cholerae isolates from four cities of Iran, were sought. Moreover the isolated $V$. cholerae from infected patients were phenotypically characterized and the presence of hemolysin gene ( $h l y A$ ) which codes for a thermostable hemolysin, an important virulence factor, was studied.

\section{Patients and Methods}

\subsection{Bactertial Isolation and Serotyping}

One hundred $V$. cholerae were isolated during 20022003 from different cities of Iran including Tehran (in the North, 32 isolates), Kermanshah (in the West, 12 isolates), Ahvaz (in the South, 30 isolates) and Kashan (in the Center, 26 isolates).The specimens were collected on sterile swabs, placed in Cary-Blair transport medium , then were immediately transferred to alkaline peptone water (APW, pH 8.6) and incubated for 6 h at temperature of 37C. They were then cultured on thiosulfate-citratebile salts-sucrose (TCBS) agar medium (Difco, Sparks, MD USA) (2).

After growth of bacteria at $37^{\circ} \mathrm{C}$ for $24 \mathrm{~h}$, the biochemical characteristics including triple sugar iron Agar (TSI), indole, simon citrate, methyl red, voges-proskauer, hemolytic activity on blood agar, motility, oxidase, urease tests (Difco) and inhibition by polymyxin B (50U/ml) (Difco) were studied $(2,3)$. Serological test using anti-sera O1, Ogawa, Inaba which were produced by Pasteur Institute of Iran was done on slide agglutination.

\subsection{Detection of Hemolysis}

To determine the hemolytic activity of $V$. cholerae samples isolated bacteria were grown in brain heart infusion broth (Difco) for $3 \mathrm{~h}$ in $37^{\circ} \mathrm{C}$ and then transferred onto the $5 \%$ sheep blood nutrient agar plates for $24 \mathrm{~h}$ at $37^{\circ} \mathrm{C}$. The colonies were then examined for hemolysis (6).

\subsection{Antibiotic Susceptibility}

Antibiotic susceptibility test was carried out based on the standard Disk diffusion method recommended by Clinical Laboratory and Standard Institute (CLSI) on Muller Hinton

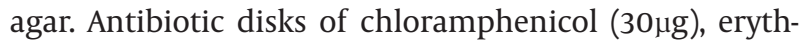

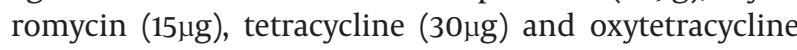

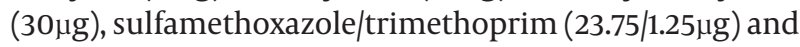
gentamycin $(10 \mu \mathrm{g})$, ciprofloxacin $(5 \mu \mathrm{g})$ and doxycycline $(30 \mu \mathrm{g})$ (BBL, Sparks, MD, USA) were used $(3,7)$.

\subsection{Polymerase Chain Reaction of hlyA}

$V$. cholera chromosomal DNA was extracted by boiling method (8) and PCR was done using the following primers to detect hlyA:

\section{Forward: 5'- GAGCCGGCATTCATCTGAAT-3'}

Reverse: 5'- CTCAGCGGGCTAATACGGTTTA-3'

under the following conditions. Initial denaturation at $94^{\circ} \mathrm{C}$ for $2 \mathrm{~min}$, followed by 25 cycles of denaturation at $94{ }^{\circ} \mathrm{C}$ for $1 \mathrm{~min}$, annealing $60{ }^{\circ} \mathrm{C}$ for $1 \mathrm{~min}$, extension at $72^{\circ} \mathrm{C}$ for $1 \mathrm{~min}$ and final extension at $72^{\circ} \mathrm{C}$ for $10 \mathrm{~min}(6$, 8).The molecular weight marker of $100 \mathrm{bp}$ and also a positive control of V. cholerae 01 El Tor ATCC 14033 were used.

\section{Results}

One hundred isolates were collected from four different cities in Iran. All samples were positive for indole, simon citrate, motility, and oxidase tests, and also they were all resistant to polymyxin $B$. Moreover, they were all negative for urease and 3 and 54\% were positive for methyl red and voges-proskauer, respectively.

Amongst the strains, 74, 23 and 3\% were considered as Ogawa, NAG (Non-agglutinable) and Inaba. All isolates from Tehran and Kermanshah, and 90 and 10\% of isolates from Ahvaz were serotyped as Ogawa and Inaba, respectively. Twenty six isolates from Kashan were NAG 88\% and Ogawa 12\% serotypes (Table 1).

Table 1. Serotyping of V.cholerae El Tor Isolated in Four Different Cities of $\operatorname{Iran}(2002-2003)$.

\begin{tabular}{llll}
\hline & Ogawa,\% & Inaba,\% & NAG,\% \\
\hline Tehran & 32 & - & - \\
Kermanshah & 12 & - & - \\
Kashan & 3 & - & 23 \\
Ahvaz & 27 & 3 & - \\
\hline
\end{tabular}

Ninety five percent of the strains were positive for $\beta$-hemolysin and were identified as Ogawa (70\%), NAG (22\%) and Inaba (3\%). Out of 5 isolates which showed negative hemolysis, 4 and 1 were Ogawa and NAG, respectively. The results from antibiotic resistance indicated that average nationwide resistances were $74,64,62,50$ and $27 \%$ of the isolates to sulfamethoxazole/trimethoprim, erythromycin, oxytracycline, tetracycline, and chloramphenicol, respectively. In Kashan, $84 \%$ of the isolates were resistant to erythromycin whereas the average resistant to this antibiotic nationwide in Iran was 64\% (Table 2). 


\begin{tabular}{|c|c|c|c|c|c|c|c|c|c|}
\hline & \multirow[b]{2}{*}{ V.Cholrea Isolated, No. } & \multicolumn{8}{|c|}{ The Percentages of Antibiotic-Resistance } \\
\hline & & Sxt & Oxy & Te & $\mathbf{E}$ & C & Dox & Gm & Cip \\
\hline Tehran & 32 & 100 & 89 & 98 & 50 & 32 & 5 & 0 & 0 \\
\hline Kermanshah & 12 & 36 & 60 & 0 & 59 & 54 & 0 & 0 & 0 \\
\hline Kashan & 26 & 0 & 0 & 0 & 84 & 0 & 0 & 0 & 0 \\
\hline Ahvaz & 30 & 100 & 100 & 100 & 60 & 23 & 10 & 8 & 0 \\
\hline
\end{tabular}

Abbreviations: C, Chloramphenicol; Cip, Ciprofloxacin; Dox, Doxcycline; E, Erythromycin; Gm, Gentamicin; Oxy, Oxytetracyclin; Sxt, Trimethoprim-Sulfamethoxazole; Te, Tetracycline

Non-hemolytic Ogawa (4 isolates) and NAG (1 isolate) showed multiple resistances to sulfamethoxazole/trimethoprim, oxytetracycline, erythromycin and tetracycline. Molecular detection of PCR indicated that all isolates were hemolysin positive (Figure 1), yet 95\% of the isolates showed hemolysin phenotype on the blood agar.

Figure 1. PCR Analysis of V.cholerae Genes in Patients Isolates and Simultaneous Use of Bacteria Staphylococcus aureus, Escherichia coli ,Aeromonas hydrophila, as Negative Controls and (V.cholerae 01 El Tor, ATCC: 14033) as a Positive Control, a Molecular Weight Marker 100 bp, Amplified Product of the hlyA Gene is $481 \mathrm{bp}$.

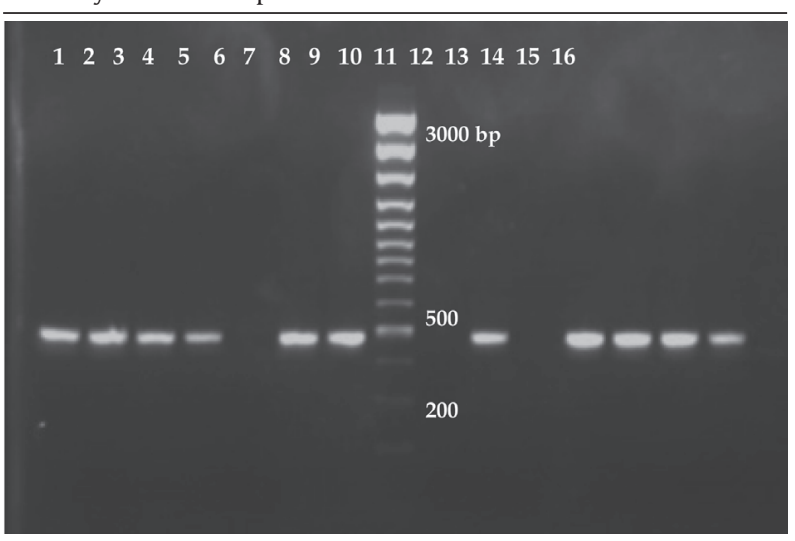

1, 2, 3, 4, 6, 7, 10, 12, 13, 14: V.cholerae,5: S.aureus,9: E.coli, 11: A.hydrophila, 15: positive control, 16: negative control.

\section{Discussion}

The present study was performed on 100 strains of $V$. cholerae El Tor collected from different of Iran. It, it was found that $95 \%$ of the strains showed hemolysis on blood agar medium, whereas all were positive PCR for hylA gene. The hlyA gene is a pathogenic factor involved in the cholera disease process, causing host tissue damage and releasing a variety of cellular components, Unlike non-hemolytic strains of the classical biotype, the El Tor strains can produce and secrete hemolysin toxin into the medium $(6,9,10)$. This is in accordance with other reports that non-hemolysin producing El Tor may be found amongst the $V$. cholerae isolates (8).

Comparing the present study results with the other published reports (6) indicated that the ratio of non-hemolytic to hemolytic isolates could vary from 5\% to $15 \%$. On the other hand, it has been reported in Latin America that V.cholerae carrying hemolysin characteristics but lacking toxin genes such as $c t x, z o t, t d h, t c p$ may still cause epidemic (11).

In the present study, 74, 23 and 3\% of all isolates were Ogawa, NAG and Inaba serotypes, suggesting that the Ogawa serotype was the predominant serotype in 2002. However, in the early 1990, the predominant serotype in Iran was Inaba (12), indicating a serotype switch from Inaba to Ogawa which still persists in Iran. Multiple antibiotic resistances to chloramphenicol (27\%), tetracycline (50\%), oxytetracycline (62\%), erythromycin (64\%) and sulfamethoxazole/ trimethoprim (74\%) were observed in the current study. Various studies in Iran and elsewhere have reported resistance of $V$. cholerae to chloramphenicol, erythromycin, kanamycin, tetracycline and sulfamethoxazole/trimethoprim $(7,13)$. Results of antibiotic susceptibility testing in 2005 in Iran showed that 86, 84, 84 and $82 \%$ of isolates were resistant to streptomycin, chloramphenicol, sulfamethoxazole/trimethoprim and tetracycline, respectively (7). Comparing the results of the two studies in different periods indicates significant drug resistant of $V$. choerlae isolates obtained in 2002 and 2005 in Iran. Such increase in antibiotic resistance among the $V$. choerlae isolates is alarming, and may indicate that constant monitoring of the drug susceptibility must be conducted.

The study from 1991 to 1994 in Angola showed multiple antibiotic resistances of $V$. cholerae to chloramphenicol, tetracycline, erythromycin and co-trimoxazole. This antibiotic resistance clone remained stable during the 4-year epidemic period in Angola (14). Collectively, these studies suggest that the multiple antibiotic resistant strains of $V$. cholerae are highly stable and may remain endemic regardless of geography. The resistance patterns in this study, indicate that ciprofloxacin for the treatment phase, and then gentamicin and doxycycline are recommended.

In this study, multiple resistance to sulfamethoxazole/ trimethoprim, oxytetracycline, erythromycin, tetracycline and chloramphenicol were found among $V$. cholerae isolates in Tehran, Kermanshah and Ahvaz. On the other hand in Kashan, except all of the isolates were susceptible to all antibiotics examined, except erythromycin. Such difference in antibiotic susceptibility pattern among the isolates could be due to eco-system of different provinces and/ or extensive use of antibiotics in Iran. Kashan as an arid 
city could affect the existence of $V$. cholerae with certain phenotypic and genotypic characteristics which needs to be studied. Therefore Monitoring serotype switching, antibiotic resistance and presence of toxin and virulence factors in $V$. cholerae is essential in limiting the dissemination of cholera at the local, regional and international levels.

\section{Acknowledgements}

We are thankful to the staff of Microbiology Dept. of Pasteur Institute of Iran for supporting in this study.

\section{Financial Disclosure}

None declared.

\section{Finding/Support}

Part of this research was financially supported by the Pasteur Institute of Iran.

\section{Authors' Contribution}

None declared.

\section{References}

1. Chhotray GP, Pal BB, Khuntia HK, Chowdhury NR, Chakraborty $\mathrm{S}$, Yamasaki S, et al. Incidence and molecular analysis of Vibrio cholerae associated with cholera outbreak subsequent to the super cyclone in Orissa, India. Epidemiol Infect. 2002;128(2):131-8.

2. Kaper JB, Morris JG, Levine MM. Cholera. Clin Microbiol Rev. 1995;8(1):48-86.

3. Tran HD, Alam M, Trung NV, Kinh NV, Nguyen HH, Pham VC, et al. Multi-drug resistant Vibrio cholerae O1 variant El Tor isolated in northern Vietnam between 2007 and 2010. J Med Microbiol. 2012;61(Pt 3):431-7.
4. Izadi S, Shakeri H, Roham P, Sheikhzadeh K. Cholera outbreak in southeast of iran: routes of transmission in the situation of good primary health care services and poor individual hygienic practices. Jpn J Infect Dis. 2006;59(3):174-8.

5. Khazaei HA, Rezaei N, Bagheri GR, Moin AA. A six-year study on Vibrio cholerae in southeastern Iran.Jpn J Infect Dis. 2005;58(1):8-10.

6. Singh DV, Matte MH, Matte GR, Jiang S, Sabeena F, Shukla BN, et al Molecular analysis of Vibrio cholerae O1, O139, non-O1, and nonO139 strains: clonal relationships between clinical and environmental isolates. Appl Environ Microbiol. 2001;67(2):910-21.

7. Pourshafie MR, Bakhshi B, Ranjbar R, Sedaghat M, Sadeghifard $\mathrm{N}$, Zaemi Yazdi J, et al. Dissemination of a single Vibrio cholerae clone in cholera outbreaks during 2005 in Iran. J Med Microbiol. 2007;56(Pt 12):1615-9.

8. Bag PK, Bhowmik P, Hajra TK, Ramamurthy T, Sarkar P, Majumder M, et al. Putative virulence traits and pathogenicity of Vibrio cholerae non-O1, non-O139 isolates from surface waters in Kolkata, India. Appl Environmen Microbiol. 2008;74(18):5635-44.

9. Pal S, Guhathakurta B, Sasmal D, Mallick R, Datta A. Purification and characterisation of a hemolysin with phospholipase C activity from Vibrio cholerae O139. FEMS Microbiol Lett.1997;147(1):115-20.

10. Vidal JE, Enriquez-Rincon F, Giono-Cerezo S, Ribas-Aparicio RM, Figueroa-Arredondo P. Culture supernatants from V. cholerae $\mathrm{O}$ El Tor strains isolated from different geographic areas induce cell vacuolation and cytotoxicity. Salud Publica Mex. 2009;51(1):39-47.

11. Colombo MM, Mastrandrea S, Leite F, Santona A, Uzzau S, Rappelli P, et al. Tracking of clinical and environmental Vibrio cholerae O1 strains by combined analysis of the presence of toxin cassette, plasmid content and ERIC PCR. FEMS Immunol Med Microbiol. 1997;19(1):33-45.

12. Pourshafie M, Grimont F, Kohestani S, Grimont PA. A molecular and phenotypic study of Vibrio cholerae in Iran.J Med Microbiol. 2002;51(5):392-8.

13. Kitaoka M, Miyata ST, Unterweger D, Pukatzki S. Antibiotic resistance mechanisms of Vibrio cholerae. J Med Microbiol. 2011;60(Pt 4):397-407.

14. Ceccarelli D, Salvia AM, Sami J, Cappuccinelli P, Colombo MM. New cluster of plasmid-located class 1 integrons in Vibrio cholerae $\mathrm{O} 1$ and a dfrA15 cassette-containing integron in Vibrio parahaemolyticus isolated in Angola. Antimicrob Agents Chemother. 2006;50(7):2493-9. 